\title{
L'expatriation du sujet. Entre nostalgie de la langue des origines et quête de l'étranger
}

\author{
In Figures de la psychanalyse, La psychanalyse et les mondes contemporains, \\ $\mathrm{n}^{\circ} 30,2015$, pp.215-219, Eres, Ramonville-Ste-Agne.
}

\section{Élise Pestre}

Dans la clinique analytique conduite auprès de patients migrants, les incidences du déplacement donnent à saisir, dans le vif du sujet, sa division. Et quand bien même cette migration s'est avérée "forcée », c'est-à-dire prescrite depuis la scène extérieure (conflits armés, catastrophes écologiques, etc.), la présence de fantasmes de mobilité préexistants au déplacement, et liés au désir du sujet, n'est pas exclue. À l'inverse, quand elle a été « souhaitée », la migration peut se révéler très angoissante une fois «consommée ». D'une certaine façon, c'est ce que formule Nancy Huston lorsqu'elle écrit qu' « un exil peut en cacher un autre ${ }^{1} »$. C'est fréquemment dans l'après-coup de l'installation en terre étrangère que ces motifs latents se révèlent, voire éclatent au grand jour, au lieu même de la subjectivité, par la production singulière de symptômes.

En contexte migratoire, le moi, «voile de cette division du sujet ${ }^{2}$ », apparaît surexposé et mis en demeure de composer avec la nouvelle réalité extérieure. Il aura pour tâche première d'imposer au réservoir pulsionnel gonflé par le déplacement, le principe de réalité. Selon la métaphore freudienne, et tel «un cavalier», il devra «réfréner la force supérieure de son cheval ${ }^{3} \gg$ devenu fougueux.

Dans ce contexte migratoire, «les restes intraduits ${ }^{4} »$ de l'inconscient refont plus facilement surface et acquièrent le pouvoir de détricoter le tissage qui existait - plus ou moins résistant - entre langue, verbe, lieu et corps, ébranlant ainsi en profondeur le parlêtre, tel que Lacan le désigne. Avec l'expatriation, qui implique la sortie «du pays où on est né ${ }^{5}$ », psychisme et corps seront souvent atteints de violentes secousses.

Le rapport du sujet expatrié à la langue dite maternelle et à celle dite d'adoption, donnera à voir, en particulier, les effets du déplacement sur sa subjectivité. Lorsque le sujet se sent menacé par la perte fantasmée de sa langue maternelle, celui-ci s'engage dans une destinée névrotique, dont nous développerons les nouages à travers une clinique de l'expatriation ${ }^{6}$. Nous verrons qu'en terre d'exil, le rapport d'aliénation du sujet à l'Autre primordial apparaît comme actualisé et exacerbé par l'écart spatial et temporel qui s'instaure.

\section{À la recherche d'une communauté de langue}

\footnotetext{
Élise Pestre, psychanalyste, maître de conférences à Paris 7, Université Paris Sorbonne Cité (USPC)

${ }^{1}$ N. Huston, Nord perdu (1998), Paris, Babel, 1999, p. 24.

${ }^{2}$ A. Vanier, Lacan, Paris, Les Belles lettres, coll. «Figures du savoir », 1998, p. 43.

${ }^{3}$ S. Freud, Le moi et le ça, (1923), Paris, Payot et Rivages, coll. « Petite bibliothèque Payot », 2010.

${ }^{4}$ Freud, (1896 a), Lettre 46, La naissance de la psychanalyse, 1969, Paris, PUF, pp.143-148, p.145.

${ }^{5}$ Expatria signifie « hors de, loin de la patrie »; soulignons que patrie est dérivée de pater, qui signifie père. A. Rey (sous la dir. de), Le grand Robert de la langue française, Paris, Dictionnaire Le Robert, 2001, p. 353.

${ }^{6}$ Cette formulation introduit une déclinaison à la «clinique de l'exil », paradigme clinique créé dans les années 1990 par F. Benslama, O. Douville, A. Cherki, R. Stitou. La clinique de l'expatriation, tout comme la clinique de l'asile (développée par É. Pestre, La vie psychique des réfugiés, Paris, Payot et Rivages, 2010) propose de mettre l'accent sur certains procédés psychiques qui apparaissent particulièrement à vif lors de l'éloignement de la terra patria.
} 
Femme française expatriée d'une quarantaine d'années, Sophie vint me consulter alors que je résidais dans une mégalopole latino-américaine, où j'étais installée comme psychanalyste. Elle me fit d'emblée état de son symptôme : «Mes enfants ne me parlent pas français. Alors je recherche un interlocuteur pour parler $m a$ langue ». Sa demande s'exprimait par le choix de son analyste dans lequel était clairement impliquée la nécessité chez elle d'une « mise en jeu de cette communauté de langue et d'une commune expérience de l'exil ${ }^{7}$ ».

Depuis son installation à l'étranger, elle avait établi peu de liens d'amitié et se sentait « loin de tout ». Initialement, il s'agissait d'un voyage touristique, puis elle y avait rencontré celui qui était devenu son époux. Ils s'étaient mariés et avaient eu deux enfants. Le couple vivait très confortablement et Sophie ne travaillait pas.

Dès notre première rencontre, la patiente évoqua ses sentiments de tristesse, d'isolement et le fait qu'elle se sentait «saturée des autochtones », des enfants en général, et des siens en particulier, trait symptomatique qu'elle désignait par «phobie des enfants ». Une agressivité que Sophie ne parvenait plus à masquer se dirigeait à l'encontre de tous ceux qui parlaient la langue du pays dans lequel elle vivait, et se cristallisait plus encore dans son lien à ses enfants bilingues et à son mari autochtone, témoignant combien le «langage n'est pas seulement communication ${ }^{8} »$, mais qu'il fonde le rapport à l'altérité, et aux semblables. Enfin, elle mentionna des troubles somatiques: des douleurs intestinales, musculaires et dorsales lancinantes qui l'amenaient à s'auto-médicamenter pour réduire ces maux qu'elle identifiait comme «tensions ».

Le lien transférentiel s'instaura sous les auspices d'un idiome commun et la question du rapport à la langue constitua le fil d'Ariane du travail analytique engagé. Ce partage des langues - maternelle et étrangère -, et de nos vies expatriées, favorisa des mouvements identificatoires et imaginaires complexes, de part et d'autre, mettant à l'épreuve la «neutralité culturelle » chère à G. Devereux, qui devrait être, selon lui, analogue à la neutralité affective qu'on attend de la situation analytique ${ }^{9}$. Ce travail s'interrompit à la suite de trois années, à l'occasion de mon propre retour en France.

\section{Parlez-moi français !}

Sophie se plaignait de manière récurrente de ses enfants qui «ne daignaient pas » lui parler français. Ce manque d'adresse dans «sa » langue était vécu tel un affront et rendu responsable de son isolement linguistique et de ses impasses relationnelles ${ }^{10}$. L'injonction au «Parlez-moi français! » apparaissait tel un cri d'amour désespéré signant l'exil de sa langue, et témoignait aussi de l'excès d'étrangeté qui l'assiégeait. D'ailleurs, la patiente en arrivait à faire du chantage à ses enfants, et à les menacer s'ils ne parlaient pas français avec elle ${ }^{11}$.

Sophie semblait redouter que sa langue maternelle lui soit arrachée, qu'elle s'évanouisse à jamais et qu'elle-même soit non seulement entamée, mais possiblement détruite par cette disparition. "La crainte de perdre la langue », dont parle Aharon Appelfeld dans un tout autre contexte d'exil, - celui de la guerre et de la Shoah -, semblait ici faire écho

\footnotetext{
${ }^{7}$ J. Hassoun, L'exil de la langue, Fragments de langue maternelle, Paris, Points Hors ligne, 1999, p. 28.

${ }^{8}$ J. Lacan, Le Séminaire, Livre XX, Encore (1972-1973), Paris, Poche, Points Essais, 1999, p. 176.

${ }^{9}$ G. Devereux, Essai d'ethno psy générale, Paris, Tel Gallimard, 1970, p. 97.

${ }^{10}$ Sophie s'adressait à ses enfants dans sa langue maternelle, selon le principe de Ronjat, actuellement recommandé par les professionnels de l'éducation où un locuteur est associé à une langue, et mis en œuvre aujourd'hui par de nombreux couples « mixtes » ayant des enfants (C. Hagege, L'enfant aux deux langues, Paris, Odile Jacob, 2005).

11 Sophie me relata une scène qui s'était produite à l'occasion d'un trajet en voiture où ses enfants jouaient bruyamment et «baragouinaient en espagnol ». Elle leur avait dit que s'ils ne se mettaient pas immédiatement à parler français elle les «attacherait avec un collier électrique qui envoie des décharges, comme ceux des chiens ».
} 
au fantasme de Sophie, quand l'écrivain rescapé énonce qu' « un homme qui perd sa langue maternelle est un infirme pour la vie ${ }^{12} »$.

Comme expatriée de sa langue, Sophie plaçait ses enfants en lieu et place de sa nécessité d'un retour à l'origine, pour tenter de reformer un rapport idéalisé à l'objet perdu. La question d'un attachement très puissant aux ascendants, et à leur langue, se posait chez elle de manière aiguë, « comme si l'expérience du toujours et de la fidélité à l'autre comme à soi supposait la fidélité indéfectible à la langue ${ }^{13} »$.

\section{La nostalgie de la langue des origines}

Le sujet s'expose violemment au retour incompressible des signifiants - parfois douloureux - de l'enfance, et des figures archaïques qui s'y rattachent lorsqu'il tente de s'en extraire par la distance territoriale de la langue première. "Ce mouvement d'aller loin, de s'égarer dans la langue de l'autre ne suffit pas pour quitter radicalement la sienne. $\mathrm{Au}$ contraire : l'aller loin dans l'étrangère expose au retour violent, panique, dans la familière ${ }^{14}{ }^{\prime}$, énonce Edmundo Gomez Mango au sujet de cet élan subjectif aux effets paradoxaux. Alors même que le désir de quitter la terre première sous-tend souvent un vœu inconscient de séparation des objets premiers qui s'y rattachent, c'est plus le ravivement de l'aliénation primaire que son extinction, que rencontre le sujet exilé à l'étranger.

Certains expatriés fantasment non seulement ce retour à la terre des origines, mais apparaissent en outre captés par une nostalgie envahissante émanant du rapport à la langue maternelle, à la lalangue, comme la désigne Lacan. Cette formulation rend bien compte de la lallation en jeu, qui en compose les sonorités mêmes. Tout comme le lait ingurgité par le nourrisson, le chant de la mère va être ingéré, et sa musicalité absorbée à jamais. Avec le vœu inconscient de retour vers cette langue ombilicale, il y aurait bien le fantasme de regagner la langue détentrice «d'un savoir absolu » dans laquelle « on pourrait tout dire, tout savoir, être totalement compris ${ }^{15} \gg$. Téter, à nouveau.

En tant qu'elle est dépositaire du refoulement et des affects primaires, la lalangue « nous affecte d'abord par tout ce qu'elle comporte comme effets qui sont affects ${ }^{16}$ », énonce Lacan. Ces affects énigmatiques, ces perceptions sensorielles «sont ce qui résulte de la présence de lalangue, en tant que de savoir elle articule des choses qui vont beaucoup plus loin que ce que l'être parlant supporte de savoir énoncé ${ }^{17} »$. La clinique des sujets expatriés rend bien compte de la façon dont le corps est fréquemment touché, parfois même altéré, par les affects primitifs qui semblent précipités par les reviviscences de lalangue, suscitées par l'environnement linguistique nouveau. D'ailleurs, les somatisations, ou autres blessures inguérissables sont répandues chez ces sujets, et donnent à voir la langue comme étant étroitement «chevillée au corps ${ }^{18}$ », le symptôme prenant corps au lieu même de la langue.

\section{La précipitation des identifications premières en terre d'exil}

Signifiants primordiaux et liens de filiation - réels et imaginaires -, sont plus que jamais ravivés lors d'une maternité d'exil. La naissance d'un enfant plonge en effet la femme

\footnotetext{
12 Témoignage d'Aharon Appelfeld, D'une langue à l'autre, film de Nurith Aviv, Swan Production, ZDF//ARTE, 55mns, 2004.

${ }^{13}$ J. Derrida, Le monolinguisme de l'autre ou la prothèse d'origine, Paris, Galilée, 1996.

${ }^{14}$ E. Gomez Mango, « La langue et l'exil », Cahiers Intersignes n ${ }^{\circ} 3$, « Parcours d'exil », 1991, p. 132.

${ }^{15}$ M. Vierling-Weiss, «Que reste-t-il ? La langue maternelle », Che vuoi ?, n 26, 2006/2, p. 17.

${ }^{16}$ J. Lacan, Encore, op. cit., p. 175.

${ }^{17}$ Ibid, p. 175.

${ }^{18}$ M. Vierling-Weiss, Ibid, op. cit., p. 5.
} 
dans son propre passé primitif, enseveli, et combien même le dépassement de la jouissance originaire s'est produit par l'entrée dans le langage, le vœu inconscient de retour nostalgique vers ce lieu archaïque, indifférencié, produit une sorte d'attraction puissante.

En effet, en devenant mère « une femme [...] est toujours convoquée à repasser par les signifiants de sa propre mère, ou encore à réutiliser pour elle, et pas forcément pour son enfant, les mots et la musique de sa langue maternelle (dans tous les sens du terme) ${ }^{19}{ }$. Sophie posa d'ailleurs les naissances de ses enfants comme événements révélateurs de sa souffrance liée à son expatriation. C'est à partir de la naissance de ses enfants dit-elle, notamment de celle du cadet, qu'elle commença à ne plus bien aller. Pour autant, ce lien conflictuel à son fils s'est dans le même temps configuré, "grâce » à la tension qu'il générait, tel un moyen de lutte contre des affects dépressifs envahissants.

La régression, cette contrainte de retour vers un temps antérieur, connu et familier, apparaît chez le sujet déplacé dans sa dimension topique et temporelle. Quand la réalité migratoire inonde le sujet, le moi accomplit un retour identificatoire à un objet antérieur, qui a servi de modèle dans le passé, modifiant ainsi le mode de satisfaction de la pulsion. Je pense ici à un patient exilé qui souffrait de dépression, installé en France depuis trois ans, et qui évoquait sa nécessité «d'être enroulé en boule » dans les draps de son lit, «comme un bébé », où il s'était mis à manger et à vivre la plupart de son temps. D'une autre manière, Sophie en vivant de manière retranchée dans un barrio cerrado ${ }^{20}$ s'était elle-même assignée à résidence. L'immobilité régnait dans cette maison qui s'apparentait à un refuge : elle contrôlait qu'aucun mouvement ne puisse faire irruption, comme pour maintenir l'équilibre psychique précaire qu'elle était parvenue à mettre en place (tissus de protection sur les meubles, etc.).

$\mathrm{Au}$ fil des séances, et en parlant de son impossible partage linguistique et langagier avec ses fils, Sophie s'est mise à beaucoup associer sur son propre rapport conflictuel à sa mère. Elle se rappelait, à l'occasion de séances, que cette femme avait $s a$ langue bien à elle, des formulations humiliantes qu'elle seule utilisait. Sophie dressa le portrait d'une femme autoritaire, qui régissait la maison comme un tyran. Elle n'était pas tant maltraitante envers elle, l'aînée, qu'à l'égard de ses deux fils, notamment « le petit dernier » qu'elle humiliait et battait fréquemment. Le père, alcoolique, était lui aussi redouté, quoique plus effacé, à l'ombre de la mère. Les parents, peu enclins à s'occuper des enfants, travaillaient beaucoup et très jeune Sophie fut désignée pour éduquer ses petits frères. À neuf ans, les parents se séparèrent et elle vécut quelques années avec sa grand-mère, avec qui elle connut répit et réconfort.

En proie à une forme de collage identificatoire massif au trait symptomatique de l'objet, la patiente était gagnée par une ambivalence importante envers ses propres enfants, notamment à l'égard du cadet. Elle semblait rejouer avec sa progéniture, et malgré sa stupéfaction devant cette répétition en partie identifiée, certaines scènes violentes vécues enfant se parant ainsi du symptôme maternel, qu'aucune distance spatiale n'empêchait de revenir en puissance.

\section{Le ravivement des opérations aliénation et séparation en terre étrangère}

Avec l'expatriation, les opérations d'aliénation et de séparation ${ }^{21}$ sont ravivées. La régression topique, qui marque un retour aux identifications premières, se voit précipitée par

\footnotetext{
${ }^{19}$ M. Vierling-Weiss, Ibid, p. 18.

${ }^{20}$ Les barrios cerrados (quartiers fermés en français) sont répandus en Amérique centrale et latine. Dans ces quartiers résidentiels clos et contrôlés par un service de sécurité privé, les résidents appartiennent généralement à des classes sociales moyennes ou aisées.

${ }^{21}$ J. Lacan, Le Séminaire, Livre XI, Les quatre concepts fondamentaux de la psychanalyse (1964-1965), Paris, Le Seuil, coll. « Points », 1973.
} 
le retour du refoulé, lui-même favorisé en contexte d'exil. Si le lien maternel primordial n'a pas fait l'objet d'un sevrage effectif, s'il n'est pas suffisamment marqué par la perte structurante qui institue l'ordre du désir, il ne pourra pas se constituer comme opérateur symbolique, et l'investissement du sujet dans un présent incarné, se verra barré. La séparation, plus encore lorsqu'elle est advenue de manière incomplète, est réactualisée par la distance spatio-temporelle et devient potentiellement destructrice, du fait de son insymbolisation. Comme nouvellement habitée par la jouissance de la lalangue, le sujet apparaît alors transposé dans ce lien d'aliénation à l'Autre, et interdit dans la construction d'un $c h e z-s o i^{22}$ qui lui permettrait de créer sa propre filiation sans trop d'empêchements dans les transmissions à venir et de vacillements dans les procédés identificatoires qui fondent la subjectivité de sa descendance. Dans ce contexte de suspension spatiale et géographique, où règne l'impossibilité de se créer une demeure propre, comment transmettre en effet à sa descendance un chez-soi habitable?

$\mathrm{Si}$ on retrouve chez les sujets névrosés, et chez les femmes en particulier ${ }^{23}$, cette question d'une coupure incomplète, c'est dans une clinique auprès de sujets exilés qu'on rencontre de manière récurrente, et avec éclat - du fait de cette reviviscence aiguë de l'identification primaire aliénante -, son incomplétude.

Le sentiment d'intrusion par la langue de l'autre ressenti chez Sophie, se manifeste notamment à travers sa phobie des enfants, qui rendrait compte d'un mécanisme de projection lié à cet étouffement primaire. Sa structure obsessionnelle, largement dévoilée par le déplacement, la conduit à ce type d'obsessions - sur les enfants - qu'elle qualifie elle-même de «bizarre ». On soulignera ici sur le paradoxe en jeu dans lequel sa tentative de vivre ailleurs, sa quête d'altérité, se double, dans le même temps, de l'impossibilité de son désir.

\section{Transmettre sa langue}

Rattrapée par la difficulté de se construire une demeure propre, Sophie projette sur ses enfants bilingues son propre rapport conflictuel entre les langues : la langue de la mère versus celle du père, la langue étrangère. "Tu préfères qui : ton père ou ta mère ? » incarne le choix impossible que Sophie énonça d'ailleurs tel quel un jour à ses fils. Les enfants apparaissent alors coupés en deux, entre d'un côté la «francité » de la mère et de l'autre, la langue du père $^{24}$. Ce découpage, aux résonances infantiles, signe bien l'entremêlement des mécanismes psychiques aux mécanismes linguistiques chez tout sujet.

La crispation d'un des parents sur sa langue maternelle - qu'il s'agisse de la parler « à tout prix » ou à l'inverse d'en interdire son utilisation - peut alimenter chez les enfants un rapport de plus en plus complexe, voire épidermique, à la langue qui se voit autant investie. En refusant de s'adresser à leur mère dans sa langue maternelle, les fils de Sophie ne témoignent-ils pas d'une tentative de dégagement à l'endroit de l'emprise maternelle ? En choisissant de s'exprimer dans la langue du père et du social, les enfants se trouvent hors de

\footnotetext{
${ }^{22}$ Le chez-soi caractérise ce lieu des affects, qui se réfère au connu et au familier, tout en ouvrant à la notion de construction d'un espace propre dans lequel le processus de subjectivation peut continuer de se déployer. Cette notion trouve une pertinence conceptuelle chez un auteur comme P. Baligand, en tant qu'elle l'articule à la spatialité, comme «mise en espace nécessaire au sujet» pour accéder à un lieu pour vivre. (P. Baligand, «Une chambre à soi: réflexions sur les liens entre espace et présentabilité », Revue Française de Psychanalyse, à paraître, 2015).

${ }^{23}$ N. Braunstein, La jouissance, un concept lacanien, Toulouse, érès, coll. « Point hors ligne », 2005.

${ }^{24} \mathrm{Je}$ me réfère ici au cas K., l'enfant de l'article de J.-P. Bauer «coincé » entre «francité » et « germanité » de ses parents. J.-P. Bauer, «L'enfant et les langues. À propos d’un cas de bilinguisme », Enfance, n 3/4, 1979, p. 195-215, p.198.
} 
portée de la langue de l'Autre maternel ${ }^{25}$. Ils s'écartent ainsi de l'exigence d'une langue privée, presque transgressive, qui exclut tout tiers séparateur, ayant pressenti que l'accès au monde extérieur " passe par la maîtrise de la langue du père, comme étrangère à la mère et à la limite comme inassimilable à la mère ${ }^{26}$ ". L'un des garçons épinglera d'ailleurs la demande sans fond de sa mère quand il s'écrira : "Mais c'est de la faute de papa si je parle mal français !» «L'imaginarisation de la différence des deux langues ${ }^{27}{ }^{2}$ qui se relie au complexe d'Edipe, dans sa version positive, est ici à son comble. Le lien mère-enfant, structurellement plus resserré à la suite d'une maternité expatriée qu'en terre autochtone, devra faire l'objet d'une séparation particulièrement énergique pour que la coupure soit opérante. Plus encore, lorsque ce rapport duel est renforcé par l'exclusivité du partage d'une langue, qui diffère de celle du père. Cette opposition des enfants témoigne également, à notre sens, d'un refus catégorique de prendre en charge le rapport si conflictuel et affecté de leur mère, à sa propre (terre) mère.

Avec ses enfants qui grandissent et deviennent des êtres parlants autonomes, Sophie semble être gagnée par une vision déformée d'elle-même. C'est comme si le miroir de leurs yeux ne lui réfléchissait plus une image unifiée de sa personne, mais qu'elle devenait étrangère à elle-même. Par le biais de la relation spéculaire à ses enfants, elle apparaît directement renvoyée à sa propre étrangèretée ${ }^{28}$, devenue irrecevable. La part d'étranger révélée par ses enfants qui grandissent, parlent la langue de l'autre, et se séparent d'elle, devient inassimilable. L'Unheimlich l'envahit, cette inquiétante étrangeté explorée par Freud $^{29}$, où l'étranger familier surgit, drainant avec lui un flot d'angoisse.

L'éloignement de la terre maternelle peut conduire certains sujets à connaître le Réel de l'en dehors de la patria, et risquer de les désarrimer symboliquement en les replongeant dans l'orbe maternel. Néanmoins, cet intervalle spatio-temporel est aussi celui qui ouvre aux sujets expatriés la possibilité d'un nouveau travail identificatoire. Dans ce contexte, le site de l'étranger, que délivre l'analyse, tel que le désigne Pierre Fédida, pourra engendrer une aptitude propre à «la construction autochtone des lieux ${ }^{30} »$. En effet, l'instauration du lien transférentiel et le travail de la langue qui se déploie dans l'analyse, favoriseront la création de modélisations langagières inédites, ainsi que la possibilité de vivre en terre étrangère.

\section{Le risque d'expatriation du lien social}

La difficulté de construire un chez-soi pour le sujet expatrié peut résider dans l'impossibilité d'instaurer un jeu productif entre les va-et-vient traductifs relatifs aux territoires (langagiers, affectifs, géographiques, etc.). Or (re)créer un espace intermédiaire qui sera fécondé par les différentes langues en circulation, s'avère à notre sens nécessaire à la construction d'un devenir ailleurs, subjectivé. Si le sujet s'égare dans les rets d'une coupure territoriale imaginarisée, clivée et sans passerelles langagières, c'est le Verwerfung qui le guette, impliquant une exclusion radicale du signifiant par la forclusion. Dans ces cas extrêmes, la nostalgie pourra aussi envahir démesurément son moi et la formation de

\footnotetext{
${ }^{25}$ Des études démontrent que la majorité des enfants bilingues qui évoluent dans un contexte linguistique autre que la langue du foyer ou de la langue maternelle de l'un des parents, communiquent au quotidien dans cette langue du social. Cependant le refus des enfants de s'exprimer dans la langue de la mère, comme c'est le cas ici, témoignent à notre sens d'un positionnement d'opposition significatif.

${ }^{26}$ J.-B. Bauer, « L'enfant et les langues », op. cit., p. 198.

27 Ibid.

${ }^{28}$ F. Benslama, «Épreuves de l'étranger », dans Z. Benchemsi, J. Fortineau, R. Beauroy (sous la direction de), Figures de l'Autre, étranger, en psychopathologie clinique, Paris, L'Harmattan, coll. «Psychanalyse et civilisation $», 1999$, p. 41-51.

${ }^{29}$ Heim renvoie en allemand au « foyer », à la maison, et introduit à la notion de familiarité.

${ }^{30}$ P. Fédida, Le site de l'étranger. La situation psychanalytique, Paris, Puf, Coll. « Psychopathologie », 1995.
} 
compromis, qui tâchait de satisfaire ses pulsions tout en composant avec les exigences de l'Autre, et ses idéaux, ne suffira plus. Le moi court le risque de se mélancoliser, l'ombre de l'objet tombant sur lui ${ }^{31}$. Dans ce contexte psychopathologique «la perte de l'objet est transformée en une perte du moi ». En tant qu'il se présente comme «moi corporel avant tout $^{32}$ », le corps se verra parfois sérieusement affecté, allant jusqu'à sombrer dans une douloureuse «position traumatique de l'étranger ${ }^{33} »$.

D’une manière générale, quand le sujet exilé se sent en péril narcissique, les processus primaires affluent et se mettent à régir son fonctionnement psychique. Dans ce contexte-là, le moi affaibli peut se sentir menacé et envahi par l'autre, l'autochtone, ce semblable étrangerfamilier avec lequel le sujet doit désormais cohabiter. Ce sentiment n'est pas sans rappeler l'extimité dont parlait Lacan, dans laquelle le proche côtoie le familier, et où l'intérieur et l'extérieur se voient soudainement rapprochés ${ }^{34}$.

Là, des fantasmes d'intrusion, voire d'engloutissement par la langue de l'autre peuvent se déployer. Sophie le formule sans équivoque quand elle dit qu'elle redoute d'être « absorbée par cette langue pauvre, la culture, le vulgaire, ce fouillis permanent, etc. ». La menace d'absorption par la langue étrangère conduit au déploiement de mécanismes projectifs, où les ennemis sont identifiés comme provenant de l'extérieur. L'agressivité se déplace ainsi vers le groupe «dominant», en tant que potentiel colonisateur de sa propre demeure langagière. La crispation identitaire du sujet incarne le «narcissisme des petites différences $^{35}$ » dans lequel l'agressivité se déplace sur l'environnement proche. La différence est fétichisée ${ }^{36}$, et l'étranger massifié. Ces défenses soulignent la façon dont la subjectivité constitue une zone frontalière, située à l'orée du collectif et de l'individuel. Dans les cas les plus extrêmes, les autochtones deviennent pour le sujet expatrié des hétéros inassimilables, des «tout autre que soi, inintelligible et dont 1 'humanité même peut faire question ${ }^{37}$ ». Ils endossent le rôle du persécuteur, ce barbare qu'il faut éliminer. Rappelons d'ailleurs que l'étymologie de barbare signifie «étranger» (barbarus) et que ce terme était utilisé en grec pour parler des Romains, et par extension de tous les autres peuples non grecs ${ }^{38}$. Là, pour le sujet en souffrance d'exil, les «barbares » sont bel et bien incarnés par les autochtones.

\section{Traduction et déplacements}

En terre étrangère la «pulsion traduisante ${ }^{39}$ » se voit ainsi constamment activée par l'altérité convoquante de la réalité extérieure nouvelle. Les mouvements traductifs qui se déploient, en deçà de la langue parlée, interpellent plus fondamentalement le sujet dans son rapport au langage et à ses butées face à l'intraduisible en jeu. Nous faisons l'hypothèse qu'à l'occasion du déplacement, les connexions avec les restes mnésiques, qui autrefois étaient des

\footnotetext{
${ }^{31}$ S. Freud, Deuil et mélancolie (1917), Paris, Payot et Rivages, coll. « Petite bibliothèque », 2011.

${ }^{32}$ S. Freud, Le moi et le ça (1923), Paris, Payot et Rivages, coll. « Petite bibliothèque », 2010, p. 65.

33 O. Douville, J. Galap, "Santé mentale des migrants et réfugiés en France », Encyclopédie MédicoChirurgicale, Psychiatrie, Paris, Elsevier, p. 880-A-10, 1999.

${ }^{34}$ Lacan écrit : «ce qui nous est le plus prochain, tout en nous étant extérieur. Il faudrait faire le mot extime pour désigner ce dont il s'agit ». Le Séminaire, Livre XVI, D’un Autre à l'autre (1968-1969), Paris, Le Seuil, 2006, p. 224.

${ }^{35}$ S. Freud, «Pourquoi la guerre? » (1933), Cahiers Intersignes, n 4-5, « La destruction », 1992, p. 68.

${ }^{36}$ R. Stitou, «Exil et déplacement culturel», Cliniques méditerranéennes, 2009/2 n 80, p. 267-280. DOI : $10.3917 / \mathrm{cm} .080 .0267$

${ }^{37}$ B. Cassin (sous la direction de), Vocabulaire européen des philosophies, Paris, Le Seuil, Le Robert, 2004.

${ }^{38} \mathrm{Ce}$ terme renvoie à « celui qui est étranger à telle race, à tel pays parce qu'il n'en parle pas la langue ou qu'il vit en dehors de sa civilisation $»$ (CNRTL).

${ }^{39} \mathrm{Je}$ m'inspire ici de la belle formule d'Antoine Berman dans L'épreuve de l'étranger qui parle de "pulsion du traduire », Paris, Gallimard, coll. « Tel », 1984, p. 21.
} 
perceptions, redeviennent plus facilement conscientes ${ }^{40}$. En effet, les allers-retours entre les territoires géographiques et langagiers propagent un «affleurement des matériaux inconscients vers le conscient $\gg$ qui favorise le retour du refoule ${ }^{41}$. Le déplacement territorial susciterait l'abaissement des défenses et leur réorganisation sur un mode plus archaïque, dans un premier temps tout du moins. L'inconscient qui est « un savoir, un savoir-faire avec lalangue $^{42} \gg$ se dévoile particulièrement à la suite du déplacement par la manifestation accrue de ses formations diverses (actes manqués, etc.). À l'occasion de cet état migratoire, les matériaux inconscients devront plus que jamais se décomposer et recomposer avec l'autre langue, mais aussi avec les bribes de lalangue qui font retour. Le langage, qui est avant tout constitué de lalangue est précisément « une élucubration de savoir sur lalangue » dit Lacan.

En revanche, quand la régression s'avère trop massive, et que le sujet se mélancolise, la dynamique psychique est entravée. Là, le risque devient la suspension de ce va-et-vient fécond des procédés de traduction, essentiels à l'incontournable traversée de "l'épreuve de l'étranger ${ }^{43} \gg$. Cet empêchement traductif - ou cette mise en exergue de la butée face à l'intraduisible - témoignera «d'une sorte d'usure de la force métaphorique dans la parole même ${ }^{44} »$. Les dessous inconscients du déplacement s'exprimeront, voire exulteront par la formation de symptômes, allant dans certaines configurations psychiques, jusqu'à défaire les pilotis langagiers fragiles sur lesquels s'était bâti le sujet, on l'a évoqué. C'est ici précisément, dans ces interstices relatifs aux traductions en reste, dans toute la polysémie qu'elles sous-tendent, que le travail analytique aura la possibilité de s'immiscer.

\section{Transfert de langue}

Malgré son rejet de la langue étrangère et l'impératif monolinguistique imposé par Sophie à ses proches et à elle-même, de nombreux termes issus de cette langue étrangère faisaient paradoxalement irruption dans son phrasé français. Or ce n'était pas n'importe quels signifiants qui émaillaient sa langue, mais ceux qui apparaissaient chargés d'affects, et qui étaient devenus imprononçables dans sa langue maternelle. "Je me sens desprotegida ${ }^{45}$ " disait-elle par exemple, dans un énoncé composé des deux langues. Dans ces cas-là, seule la langue étrangère semblait à même de pouvoir accueillir certains signifiants. Au fil des séances, l'oscillation entre les deux langues devint à chaque fois plus prégnante. Mes interprétations trouvèrent souvent leur genèse dans des questionnements axés sur ces signifiants que Sophie ne traduisait pas en français, donnant naissance à un va-et-vient fécond entre les langues, qui rendait compte de la reprise d'une certaine mobilité psychique. Avec le transfert, la régression engagée permit de traverser, via la langue maternelle commune, des conflits infantiles en lien avec les imagos parentales archaïques. Cette situation clinique singulière, où le partage de la langue maternelle s'ajoutait à celui de la langue étrangère, favorisa de nouveaux jeux identificatoires chez la patiente. Le lien transférentiel permit également l'expression de mouvements d'amour et de haine à la portée séparatrice.

Cet espace traductif encouragea l'avènement d'une nécessaire dialectique entre l'étranger et le familier. Et les butées face à l'intraduisible en jeu purent progressivement se délier grâce à ce lien qui favorisa chez elle un certain rapport réconcilié aux langues.

\footnotetext{
${ }^{40}$ S. Freud, Le moi et le ça, op. cit., p. 55.

${ }^{41}$ É. Pestre, « Giving Birth in Exile: Motherhood as Reterritorialization », American Journal of psychoanalysis, à paraître 2015.

${ }^{42}$ J. Lacan, Encore, op. cit, p. 175.

${ }^{43}$ A. Berman, «L'épreuve de l'étranger », op. cit.

${ }^{44}$ F. Benslama, «L'enfant et le lieu », Cahiers intersignes, n 3 , « Parcours d'exil », 1991, p. 63.

${ }^{45}$ Terme qui exprime en français le fait de se sentir déprotégée, en insécurité.
} 
Face à la menace d'une langue devenue totalitaire, celle d'une tour de Babel assourdissante, l'expérience impulsée par la rencontre avec "le site de l'étranger ${ }^{46}$ ", l'analyse, permettrait de décoller le sujet d'un fantasme de retour vers la langue originaire perdue, où l'indifférenciation règne. Ce site, «qui est celui du langage, lieu des lieux, comme lieu des localités (psychiques) ${ }^{47} \gg$, permet une (re)traversée subjectivante de la langue maternelle, passage que la rencontre avec une langue autre, étrangère, encourage déjà.

\section{Conclusion}

Lors de l'éloignement de la terra patria, les opérations primitives qui permettent la construction du psychisme du sujet sont ravivées. Avec la rencontre d'un environnement linguistique nouveau, les signifiants douloureux de l'enfance peuvent refaire violemment surface chez le sujet expatrié et le conduire à un sentiment de menace vis-à-vis de l'autochtone, et de sa langue, comme nous l'avons envisagé avec le cas de Sophie. Gagnée par une nostalgie écrasante, et à la recherche de la langue originaire perdue, la patiente apparaissait bien clivée entre deux existences et deux langues. C'est à l'orée d'un travail traductif, facilité par le site qu'offre l'analyse, que l'élaboration d'un rapport au lieu subjectivé peut advenir. Le rapport à la langue pourra dans ce contexte être régénéré, tout comme les imagos qui s'y rattachent, rendant compte de la fécondation du «propre par la médiation de l'étranger ${ }^{48} »$. La dimension métaphorisante du travail langagier qui se déploie dans l'espace transférentiel sera en mesure de médiatiser l'afflux de jouissance envahissant du retour de la lalangue, réduisant les risques d'expatriation subjective. Le sujet expatrié sera ainsi in fine en capacité de s'approprier - ou pas - ce déplacement, l'historiciser, pour l'intégrer dans sa continuité d'existence. Et c'est à cette condition qu'il pourra créer sa demeure propre, un chez-soi à lui, ailleurs, sans se sentir terrassé par la langue de l'autre.

\section{RESUME}

L'auteure propose d'explorer le rapport du sujet expatrié aux langues maternelle et étrangère. À la suite de son déplacement territorial, le sujet se sent parfois envahi par la nostalgie de la terra patria et gagné par le vœu inconscient d'un retour à la langue maternelle première, la lalangue selon la formule de Lacan. Malgré son désir de vivre ailleurs, il peut se mélancoliser, ou ressentir des sentiments de menace et d'envahissement par l'autochtone et sa langue. Ces vécus intrusifs pourront le conduire à l'impossibilité de construire un chez-soi à lui, ainsi qu'à des vacillements dans ses transmissions, comme nous l'envisagerons avec le cas de Sophie. En terre étrangère, le passage par un travail analytique permettra un va-et-vient productif entre les langues, favorisant l'avènement d'une nécessaire dialectique entre l'étranger et le familier.

MOTS-CLES : Expatriation, langue maternelle, traduction, étranger, transmission

The Expatriation of the Subject. Between Nostalgia for the Language of Origins and the Search for the Foreign".

\section{SUMMARY}

The author sets out to explore the expatriate subject's relationship to the mother tongue and to foreign language. In the wake of a territorial displacement, the subject sometimes feels invaded by nostalgia for the terra patria, and is under the influence of an unconscious wish to return to the initial mother tongue, or what Lacan called lalangue. Despite the subject's desire to live elsewhere, he or she may become melancholic, or feel threatened or invaded by the indigenous population and their language. This experience of intrusion might lead the subject to the impossibility of constructing a own sense of

\footnotetext{
${ }^{46}$ P. Fédida, Le site de l'étranger. La situation psychanalytique, op. cit.

${ }^{47}$ P. Fédida, Ibid, p. 273.

${ }^{48}$ A. Berman, «L'épreuve de l'étranger », op. cit., p.16.
} 
homeliness, along with vacillations in transmissions, as we shall see in the case of Sophie. On foreign soil, engaging in psychoanalytical work can enable a productive back and forth between languages, thereby favoring the advent of a necessary dialectic between the foreign and the familiar.

KEYWORDS: Expatriation, mother tongue, translation, foreigner, transmission. 